J. Lake Sci. ( 湖泊科学 ), 2006, 18( 1 ):43-48

http:// www. jlakes. org. E-mail: jlakes@ niglas. ac.cn

(c) 2006 by Journal of Lake Sciences

\title{
巢湖沉积物磁性特征及其对沉积动力的响应"
}

\author{
谢红霞 $^{1}$, 张卫国 ${ }^{1}$, 顾成军 ${ }^{2}$, 戴雪荣 ${ }^{2}$, 俞立中 ${ }^{1,3}$, John Dearing $^{4}$ \\ ( 1 : 华东师范大学河口海岸国家重点实验室, 上海 200062 ) \\ ( 2 : 华东师范大学地理系, 上海 200062 ) \\ ( 3 : 上海师范大学, 上海 200234 ) \\ ( 4 :Department of Geography, University of Liverpool, Liverpool L69 7ZT, UK )
}

\begin{abstract}
摘 要: 对巢湖西部柱样以及杭埠河流域土壤的环境磁学研究表明, 磁铁矿是决定磁性特征的主要磁性矿物, 杭埠河流域 是巢湖西部沉积物的主要来源. 靠近巢湖西岸的柱样 AC1 砂含量较高, $\chi$ 、SIRM 值较大而 $\chi_{\mathrm{ARM}} / \mathrm{SIRM}$ 较低, 说明磁铁矿含 量较高、颗粒较粗. 距岸稍远的柱样 AC2 砂含量较低, $\chi$ 、SIRM 值也较低, 其变化与 $\chi_{\mathrm{ARM}}$ 较相似, 与粘土含量变化也有一定 程度的相似, AC2 沉积物 $\chi$ 、SIRM 值较 AC1 小,但 $\chi_{\mathrm{ARM}} / \mathrm{SIRM}$ 较高, 说明磁铁矿含量较低且颗粒较细. 杭埠河流域土壤的 磁性测量结果表明, 砂、砾含量高的粗骨土磁性最强, 富含粗颗粒磁铁矿. 巢湖柱样沉积物砂的含量随踞岸远近而不同, 反映了沉积环境的水动力不同, 由此产生明显的磁性特征差异, 实质是对沉积动力的响应. 研究表明磁性参数 $\chi_{\mathrm{ARM}} / \mathrm{SIRM}$ 可以间接反映沉积物中粘土含量的高低变化.
\end{abstract}

关键词: 磁性特征; 粒度; 沉积动力; 巢湖

\section{Magnetic properties of sediments from Lake Chaohu and its response to sedimentary dy- namics}

XIE Hongxia $^{1}$, ZHANG Weiguo ${ }^{1}$, GU Chengjun ${ }^{2}$, DAI Xuerong ${ }^{2}$, YU Lizhong ${ }^{1,3} \&$ John Dearing ${ }^{4}$ ( 1 : State Key Laboratory of Estuarine and Coastal Research, East China Normal University, Shanghai 200062, P. R. China )

( 2:Department of Geography, East China Normal University, Shanghai 200062, P. R. China)

( 3 : Shanghai Normal University, Shanghai200234,P. R. China)

( 4:Department of Geography, University of Liverpool, Liverpool L69 7ZT, UK )

\begin{abstract}
Magnetic study on core sediments from western Lake Chaohu and soils from Hangbu River Catchment suggests that magnetite is the dominant magnetic mineral, and soils from Hangbu River Catchment are the main source of lake sediments. Core AC1 near the bank shows higher proportion of sands and higher $\chi$ 、SIRM values but lower $\chi_{\text {ARM }} /$ SIRM values, indicating higher concentration of magnetite with coarse size. Core AC2 more far away from the bank shows lower proportion of sands and lower $\chi$ SIRM values. The similar variations of $\chi$, SIRM with $\chi_{\mathrm{ARM}}$, and to a lesser degree with clay fraction, in combination with higher $\chi_{\mathrm{ARM}} / \mathrm{SIRM}$ values, indicating lower concentration of magnetite with fine size. Magnetic properties of soils in the catchments indicate that skeletal soils with abundant sand and gravel fraction are magnetic strong which are contributed by coarse magnetite. Content of sand fraction in core is a reflection of water energy which is dependent on the distance to the bank. Therefore, the distinct magnetic properties of different cores are due to sedimentary dynamics. It is found that $\chi_{\mathrm{ARM}} / \mathrm{SIRM}$ can be used to indicate the variation of clay contents in sediments.
\end{abstract}

Keywords: Magnetic properties; particle size; sedimentary dynamics; Lake Chaohu 
20 世纪 70 年代中期以来,由于磁性测量方法快速、简便、经济、非破坏性等特点,利用湖泊沉积物磁性 特征进行古环境演变研究得到了广泛的应用 ${ }^{[1-5]}$. 沉积物的磁性特征直接反映的是磁性矿物的含量、类型 和颗粒大小等信息,这些因子往往是沉积物物源、搬运和沉积动力以及沉积后次生变化等综合作用的结 果 ${ }^{[6]}$,受控于气候和人类活动,因而沉积物磁性特征蕴含了丰富的环境演变信息.

巢湖是我国五大淡水湖泊之一,近年来由于湖泊富营养化问题而备受关注 ${ }^{[7]}$,但有关利用巢湖沉积物 进行流域环境演变信息提取的研究尚不多见 ${ }^{[8,9]}$. 本文对巢湖沉积物进行了磁性特征和粒度分析,并结合 流域土壤、河流悬浮泥沙的磁性特征,着重探讨了沉积物磁性特征与沉积动力的关系.

\section{1 研究区域和方法}

巢湖目前湖泊面积约 $769 \mathrm{~km}^{2}$, 平均水深 $2.69 \mathrm{~m}$, 湖区属亚热带季风气候 ${ }^{[10]}$. 位于巢湖西部的大别山 区是巢湖泥沙的主要来源, 成土母质包括花岗岩、片麻岩、片岩、板岩等岩石和下蜀黄土 ${ }^{[7]}$. 自西南向东北 流的杭埠河是巢湖主要的输水、输沙河流. 由于泥沙淤积和围怎, 湖泊西岸不断向东迁移,湖面逐渐 收缩 ${ }^{[10]}$.

2003 年 7 月在杭埠河口采集了柱样 AC1 ( $31^{\circ} 33^{\prime} \mathrm{N}, 117^{\circ} 22^{\prime} \mathrm{E}$, 长 $\left.142 \mathrm{~cm}\right)$ 和 $\mathrm{AC} 2\left(31^{\circ} 33^{\prime} \mathrm{N} 、 117^{\circ} 24^{\prime} \mathrm{E}\right.$, 长 $96 \mathrm{~cm}$ ) (图 1), 两者相距约 600-700 m, 同时沿杭埠河自上游向下游采集了 10 个悬浮泥沙样品. 2004 年 3 月在杭埠河流域采集了表层土样 70 个. 柱样以 $1 \mathrm{~cm}$ 间隔分割,所得沉积物样品以及土壤、悬浮泥沙样 品于 $40^{\circ} \mathrm{C}$ 低温下烘干,除保留样品作粒度分析外,其余样品用研钵分散后进行磁性测量.

对所有样品进行了低频磁化率 $\left(0.47 \mathrm{kHz}, \chi_{\mathrm{LF}}\right)$ 和高频磁化率 $\left(4.7 \mathrm{kHz}, \chi_{\mathrm{HF}}\right)$ 、非磁滞剩磁磁化率 $\left(\chi_{\mathrm{ARM}}\right.$, 交 流磁场峰值为 $100 \mathrm{mT}$, 直流磁场为 $0.04 \mathrm{mT}$ )及等温剩磁( IRM, 磁场强度依次为 $1 \mathrm{~T} 、-100 \mathrm{mT}$ )测量, 所用仪器 为英国 Bartington MS2 磁化率仪、Molspin 交变退磁仪、脉冲磁化仪和 Minispin 旋转磁力仪. 本文中磁化率( 系 指 $\chi_{\mathrm{LF}}$, 将 $\mathrm{IRM}_{1 \mathrm{~T}}$ 称为饱和等温剩磁 $(\mathrm{SIRM})$, 并计算了频率磁化率 $\left(\chi_{\mathrm{fd}} \%=\left[\chi_{\mathrm{LF}}-\chi_{\mathrm{HF}}\right] / \chi_{\mathrm{LF}} \times 100\right)$ 、比值参数 $\chi_{\mathrm{ARM}} / \mathrm{SIRM}$ 和 $\mathrm{S}_{-100}\left(\left[\mathrm{SIRM}-\mathrm{IRM}_{-100 \mathrm{mT}}\right] / 2 \mathrm{SIRM} \times 100\right)$. 根据磁性测量结果, 选择了典型样品进行了热磁曲 线的测量,所用仪器为 MMVFTB 可变场磁天平. 粒度分析采用 Coulter LQ - 100Q 激光粒度仪完成.

\section{$117^{\circ} 00^{\prime} \mathrm{E}$}

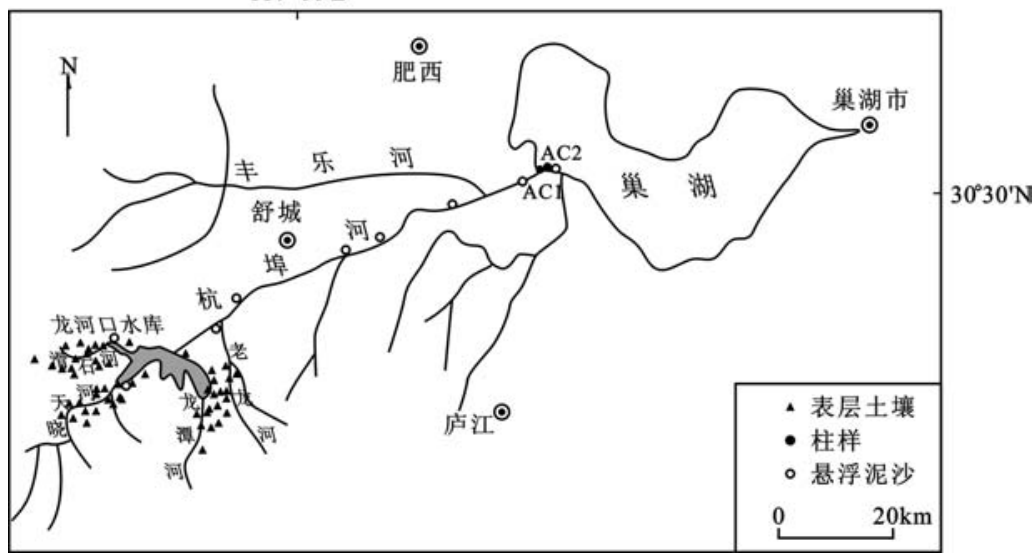

图 1 研究区域及采样站位

Fig. 1 Map of study area showing sampling sites

\section{2 研究结果与讨论}

\section{1 沉积物磁性特征}

柱样 AC1、AC2 磁性特征及粒度组成的垂向变化见图 2. Х S SIRM 通常反映了样品中磁性矿物的含量,

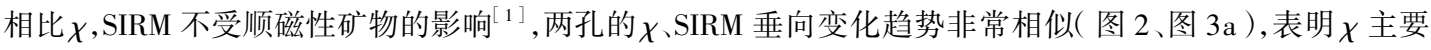


是由亚铁磁性矿物贡献. 两个柱样 $\chi$ 、SIRM 自下向上, 总体皆呈上升趋势, 但这一趋势在 AC1 $50-90 \mathrm{~cm}$ 、 AC2 40-60 cm 深度分别为一磁性较强层次所破坏, 特别是 AC1 这一深度出现了柱样中 $\chi$ 、SIRM 的最大值. 与 $\mathrm{AC} 2$ 相比, AC1 中 $\chi$ SIRM 值总体偏高, 说明了 AC1 含有较多的磁性矿物.
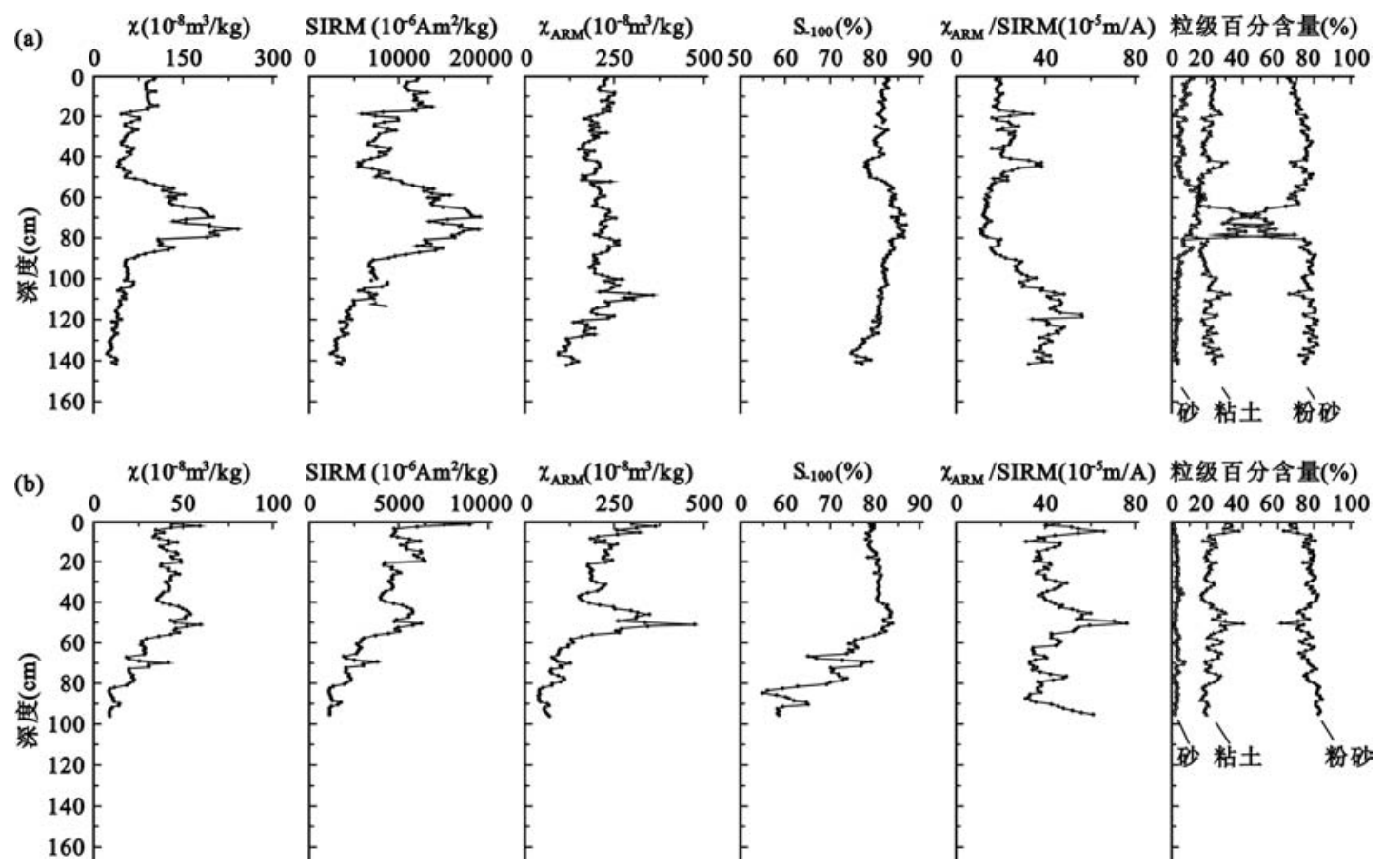

图 2 巢湖柱样 $\operatorname{AC1}(\mathrm{a}) 、 \mathrm{AC2}(\mathrm{b})$ 磁性参数及粒度组成的垂向变化

Fig. 2 Vertical variations of magnetic properties and particle size of core AC1 ( a ) and AC2( b )

典型样品 ( AC1 深度 $76 \mathrm{~cm}$ 与 AC2 深度 $1 \mathrm{~cm} 、 85 \mathrm{~cm}$ ) 的热磁曲线表明( 图 4 ), 不论样品 $\chi$ 值高低, 磁性 矿物居里温度都在 $580^{\circ}$ 左右, 说明磁铁矿是主要的磁性矿物. 所有样品的磁化强度在加热和冷却过程中都 呈现不可逆的特征. AC1 深度 $76 \mathrm{~cm}$ 与 AC2 深度 $1 \mathrm{~cm}$ 沉积物样品加热曲线显示, 自室温至 $300^{\circ} \mathrm{C}$ 附近, 磁 化强度有上升的趋势, 很可能是纤铁矿转变为磁赤铁矿所致, 而 $300^{\circ} \mathrm{C}$ 至 $450^{\circ} \mathrm{C}$ 之间, 加热曲线是下凹特征, 反映了磁赤铁矿向赤铁矿的转变 ${ }^{[1]}$.

$\chi_{\mathrm{fd}} \%$ 指示了超顺磁颗粒含量的多少 ${ }^{12]}$, 由于大部分样品的 $\chi_{\mathrm{fd}} \%$ 值一般在 $0 \%-2 \%$ 左右, 最大值不超 过 $4 \%$, 说明湖泊沉积物中超顺磁颗粒很少. $\chi_{\mathrm{ARM}}$ 对单畴颗粒( SD ) 极具敏感性 ${ }^{[12]}$. AC1 中 $\chi_{\mathrm{ARM}}$ 与 $\chi$ SIRM 的垂向变化差异较大, 特别是 50-80 $\mathrm{cm}$ 的强磁性层 $\chi_{\mathrm{ARM}}$ 并未明显增高, 说明该柱样中 $\chi 、 \mathrm{SIRM}$ 主要不是由 $\mathrm{SD}$ 颗粒贡献的. $\mathrm{AC} 2$ 中 $\chi_{\mathrm{ARM}}$ 垂向变化与 $\chi$ 、SIRM 较为相近, 说明该柱样中的 SD 颗粒对 $\chi$ 、SIRM 具有重要贡 献. $\chi_{\mathrm{ARM}} / \mathrm{SIRM}$ 可用来指示磁性颗粒大小,较高的比值指示较细的磁性颗粒 ${ }^{[2]}$. 图 2 表明 AC1 中 $\chi$ 、SIRM 高值样品一般具有较低的 $\chi_{\mathrm{ARM}} / \mathrm{SIRM}$, 即磁性强的样品, 磁性矿物颗粒较粗, 图 $3 \mathrm{~b}$ 也证实了这一点. AC2 总 体上 $\chi_{\mathrm{ARM}} / \mathrm{SIRM}$ 波动较小, 如 $60 \mathrm{~cm}$ 深度以下, 尽管沉积物 $\chi$ 值较低, 但 $\chi_{\mathrm{ARM}} / \mathrm{SIRM}$ 与 $40 \mathrm{~cm}$ 深度以上样品 相接近. 该柱样 40-60 cm 深度 $\chi$ 、SIRM 的高值层段对应了较高的 $\chi_{\mathrm{ARM}} / \mathrm{SIRM}$, 说明该层位细颗粒的磁性矿 物对磁性的增强起着重要的作用. 此外, AC2 中仍可以看出一些具有 $\chi 、 S I R M$ 高值的样品, 如深度 $70 \mathrm{~cm} 、 90$ $\mathrm{cm}$, 其 $\chi_{\mathrm{ARM}} / \mathrm{SIRM}$ 较低, 表明这些深度的沉积物含有较多的粗颗粒磁性矿物.

$\mathrm{S}_{-100}$ 是指示磁性矿物类型的指标, 它可以较灵敏的反映亚铁磁性矿物和不完整反铁磁性的相对组成, 随着不完整反铁磁性矿物的贡献的增加而下降 ${ }^{13]}$. AC1 自底部向上至 $80 \mathrm{~cm}$ 深度, $\mathrm{S}_{-100}$ 值呈增加趋势; 80 
$-50 \mathrm{~cm}$ 区间, $S_{-100}$ 值总体较高; 自 $50 \mathrm{~cm}$ 至表层, $S_{-100}$ 呈稍许上升趋势. AC2 亦有类似的变化趋势, $60 \mathrm{~cm}$ 深度以下, $S_{-100}$ 显著偏低, 向上呈上升趋势, 且较 AC1 更为显著; $40-60 \mathrm{~cm}$ 深度也是剖面 $S_{-100}$ 高值区间段, $40 \mathrm{~cm}$ 以上至表层, $\mathrm{S}_{-100}$ 变化不大. $\mathrm{S}_{-100}$ 变化趋势与 $\chi, \mathrm{SIRM}$ 总体相似, 即磁性强的样品具有较高的 $\mathrm{S}_{-100}$, 反之, 磁性较弱的样品中不完整反铁磁性矿物的贡献较为显著( 图 $3 \mathrm{c}$ ).
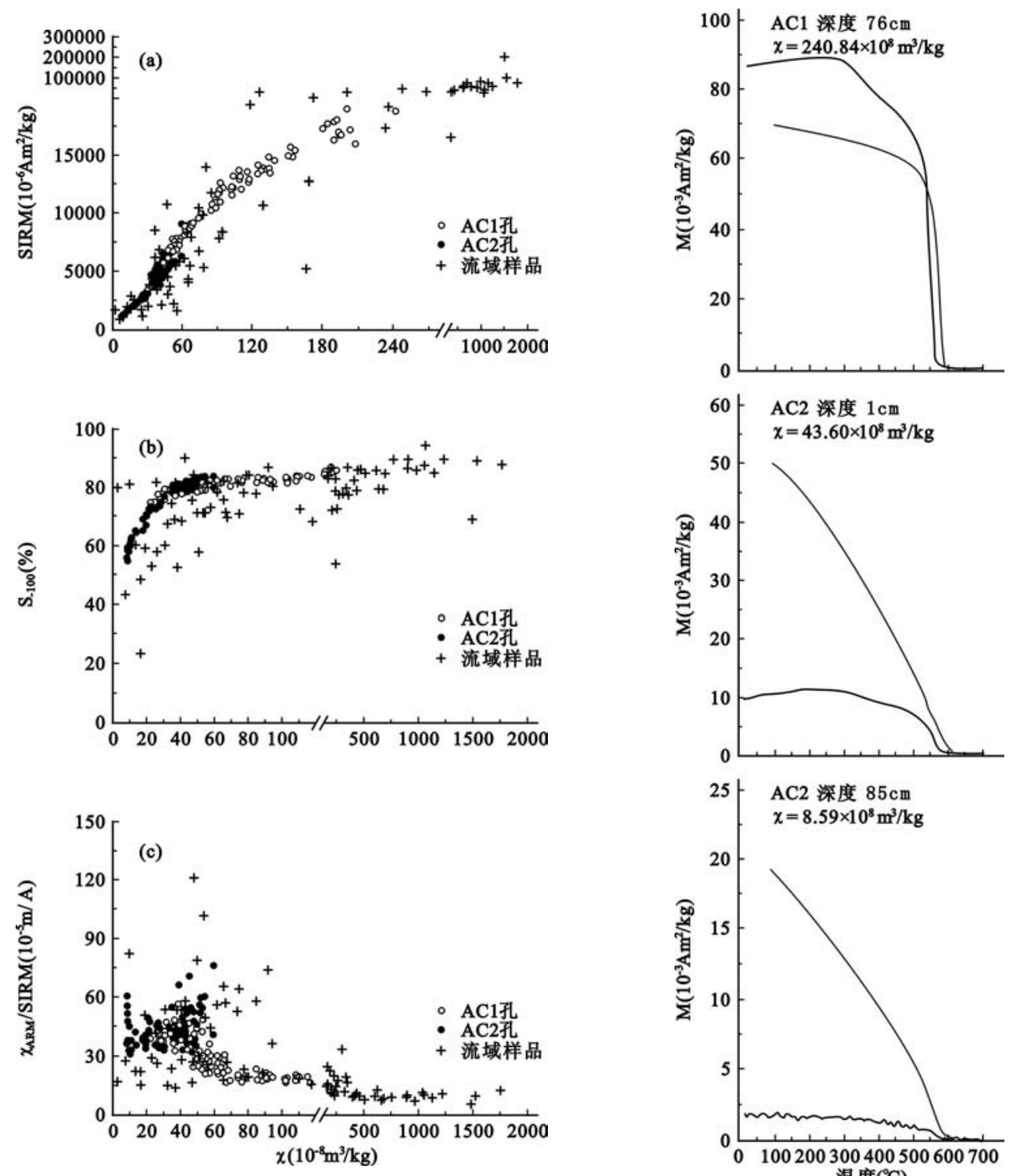

图 3 巢湖沉积物及杭埠河流域土壤 $\chi$ 与 $\operatorname{SIRM}(\mathrm{a})$, $\chi_{\mathrm{ARM}} / \operatorname{SIRM}(\mathrm{b})$ and $\mathrm{S}_{-100}(\mathrm{c})$ 关系图

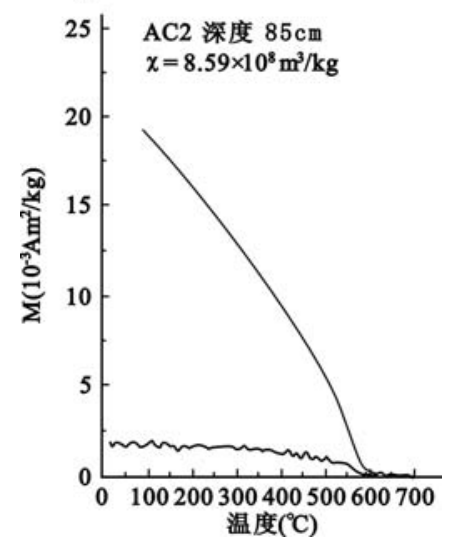

图 4 巢湖沉积物典型样品热磁曲线,

粗( 细)线表示加热( 冷却)过程

Fig. 3 Plot of c verus $\operatorname{SIRM}(\mathrm{a}), \chi_{\mathrm{ARM}} / \operatorname{SIRM}(\mathrm{b})$ and

$\mathrm{S}_{-100}(\mathrm{c})$ for Chaohu core sediments as well as soils from Hangbu River catchment

Fig. 4 Thermomagnetic curves of typical lake sediments. The bold and thin line repsents heating and cooling stages, respectively 


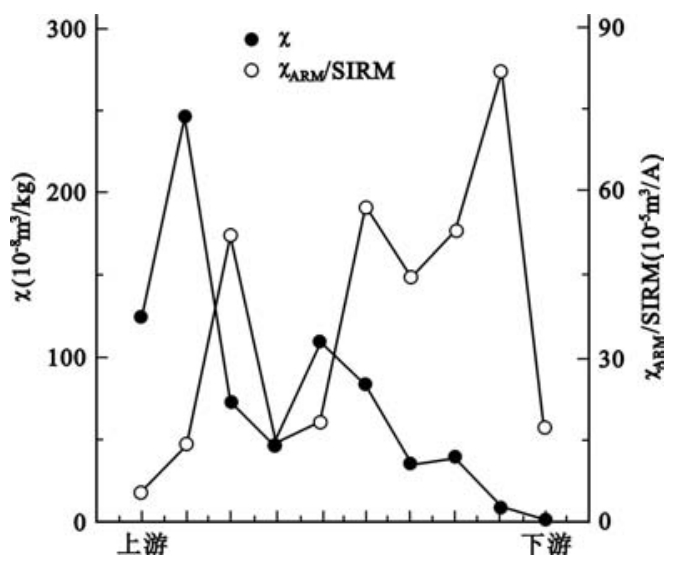

图 5 杭埠河悬浮泥沙 $\chi(\mathrm{a})$ 与 $\chi_{\mathrm{ARM}} / \operatorname{SIRM}(\mathrm{b})$ 的空间变化

Fig. 5 Variations of $\chi(\mathrm{a})$ and $\chi_{\mathrm{ARM}} / \operatorname{SIRM}(\mathrm{b})$ of suspended matters from upper stream down to lower stream of Hangbu River

\section{2 磁性特征对沉积动力的响应}

图 3 显示, 两个柱柱中具有中等磁化率 $\left(20 \times 10^{-8}-50 \times 10^{-8} \mathrm{~m}^{3} / \mathrm{kg}\right)$ 的样品出现重叠, 反映了这些样品 具有相似的磁性特征. 此外, AC1 中具有高 $\chi$ 、SIRM、 $S_{-100}$ 值和低 $\chi_{\mathrm{ARM}} / \mathrm{SIRM}$ 值的样品更多, 表明这些样品 中磁铁矿含量较多, 且颗粒较粗, 反之, $\chi$ SIRM、 $S_{-100}$ 低值及 $\chi_{\mathrm{ARM}} / \mathrm{SIRM}$ 高值样品更多出现在 AC2 中. 已有 研究表明,粒度是影响磁性特征的重要因素 ${ }^{[1,14]}$. 对比两孔的粒度组成, 可以发现 AC1 孔砂的含量较高, 且 $\chi$ 、SIRM 值与砂的含量呈同步变化, 但 $\chi_{\mathrm{ARM}} / \mathrm{SIRM}$ 却与砂的含量呈反向变化趋势, 说明磁铁矿主要富集于 砂中, 且磁铁矿颗粒较粗. AC2 中 $\chi_{\mathrm{ARM}}$ 与 $\chi$ 、SIRM 基本呈同步变化, 且 $\chi_{\mathrm{ARM}} \chi_{\mathrm{ARM}} / \mathrm{SIRM}$ 与粘土百分含量具有 较相似的垂向变化, 说明 AC2 中 $\chi$ 、SIRM 主要由粘土中含有的细颗粒磁性矿物所贡献. 很显然, 粘土中含有 的磁铁矿要低于砂.

对流域土壤磁性特征研究表明,流域土壤磁性差异较为显著, $\chi$ 变化于 $(7-2541) \times 10^{-8} \mathrm{~m}^{3} / \mathrm{kg}$ 之间. 图 3 显示, AC1 与 AC2 的沉积物磁性特征都包含在流域土壤磁性变化范围之内, 说明杭埠河流域是巢湖沉 积物的主要来源, 但巢湖沉积物的 $\chi$ 值与流域土壤的低值较为一致, $\chi$ 大于 $300 \times 10^{-8} \mathrm{~m}^{3} / \mathrm{kg}$ 的样品没有在 沉积物中出现. $\chi$ 值大于 $300 \times 10^{-8} \mathrm{~m}^{3} / \mathrm{kg}$ 的土壤样品, $\chi_{\mathrm{ARM}} / \mathrm{SIRM}$ 较低( 图 $3 \mathrm{~b}$ ), 说明强磁性样品中磁性矿 物颗粒较粗, 这些土壤的成土母质为花岗岩、片麻岩, 属于砂、砾含量高的粗骨土. 尽管粗颗粒土壤中含有 大量的粗颗粒磁铁矿, 但很难作长距离搬运, 主要在上游河道中沉积下来, 因而进入湖泊的泥沙主要是磁性 相对较弱的细颗粒泥沙. 图 5 揭示了杭埠河悬浮泥沙的磁性特征变化,自上游向下游, $\chi$ 呈现明显的减小 趋势, $\chi_{\mathrm{ARM}} / \mathrm{SIRM}$ 却呈现增加的趋势, 说明在泥沙搬运过程中, 随着水动力减弱, 泥沙中的粗颗粒磁性矿物 率先沉积, 导致泥沙中磁性矿物含量下降和磁性矿物颗粒变细.

由于 AC1 靠近巢湖杭埠河口, 能够堆积更多的粗颗粒泥沙, 因而 AC1 磁性较强,但磁性矿物颗粒较粗. 从垂向上看, AC1 沉积物的粒度, 如不考虑 80-50 cm 深度, 存在随深度变浅, 总体变粗的现象. 由于巢湖西 岸不断向东推进 ${ }^{[10]}$, 因此上述粒度变化反映了湖泊进积过程中, AC1 距岸距离缩短的过程. 沉积物的磁性 参数变化也响应了这样一个过程, 体现在磁性矿物含量上升和颗粒变粗上. 值得注意的是, AC1 中 $80-50$ $\mathrm{cm}$ 深度的沉积物粒度急剧粗化破坏了这一趋势, 很可能指示了流域异常大的洪水事件, 水动力陡然增强, 致使流域内侵蚀加剧, 大量粗颗粒泥沙被带入湖泊, 从而使得沉积物的磁性也突然增强. AC2 由于距岸较 远, 砂的含量较低, 沉积物相应磁性较弱, $\chi$ SIRM 大小主要由粘土含量决定. 可以看出, 在两个柱样中, $\chi_{\mathrm{ARM}} / \mathrm{SIRM}$ 的高值指示了沉积物中粘土含量较高的层次, 反之则是砂含量较高的层次. 如 AC2 中 $90 \mathrm{~cm} 、 70$ $\mathrm{cm}$ 深度, 其较高的 $\chi$ SIRM 具有较低的 $\chi_{\mathrm{ARM}} / \mathrm{SIRM}$, 说明是由粗颗粒的磁铁矿所贡献的,相应的这些层位沉 积物中砂的含量也较高. 同样, AC1 中某些砂含量较低但粘土含量较高的层位, 如 $19 \mathrm{~cm} 、 42-45 \mathrm{~cm}$ 以及 
$110 \mathrm{~cm}$ 以下, $\chi$ 、SIRM 较低但 $\chi_{\mathrm{ARM}} / \mathrm{SIRM}$ 较高, 说明这些深度沉积物中细颗粒磁铁矿的贡献非常显著.

\section{3 结论}

巢湖西部沉积物的环境磁学研究表明, 磁铁矿是决定磁性特征的主要磁性矿物, 杭埠河流域是巢湖西 部沉积物的主要来源. 由于杭埠河流域砂、砾含量高的粗骨土中富含粗颗粒磁铁矿, 总体上砂含量高的沉 积物磁铁矿含量较高而颗粒较粗, 粘土含量高的沉积物磁铁矿含量较低且颗粒较细, 且不完整反铁磁性矿 物对磁性特征的贡献更为显著. 巢湖柱样随踞岸远近不同而显示出明显的磁性特征差异, 是沉积动力环境 不同所致,磁性参数 $\chi_{\mathrm{ARM}} / \mathrm{SIRM}$ 可以间接反映沉积物中粘土含量的高低变化.

\section{4 参考文献}

[1] Thompson R, Oldfield F. Environmental Magnetism. London: Allen and Unwin,1986:1 - 227.

[2] 胡守云, 王苏民, Appel E 等. 呼伦湖湖泊沉积物磁化率变化的环境磁学机制. 中国科学( D 辑 ), 1998, 28( 4$): 334-339$.

[3] 朱立平,王君波,陈 玲等. 藏南沉错湖泊沉积多指标揭示的 2 万年以来环境变化. 地理学报, 2004, 59( 4$): 514-524$.

[4] 汪卫国,冯兆东, 李心清等. 蒙古北部 Gun Nuur 湖记录的全新世气候突发事件. 科学通报,2004,49 ( 1 ):27 - 33 .

[ 5 ] Dearing J A, Hu Y Q, Doody P et al. Preliminary reconstruction of sediment-source linkages for the past 6000 yrs at the Petit Lac d'Annecy, France, based on mineral magnetic data. Journal of Paleolimnology, $2001,25: 245-258$.

[6] Oldfied F. Environmental magnetism - a personal perspective. Quaternary Science Reviews, 1991, 10:73 85.

[7] 屠清瑛, 顾丁锡, 尹澄清等. 巢湖富营养化的研究. 合肥: 中国科学技术大学出版社, 1991:1-114.

[8] 姚书春,沈 吉. 巢湖沉积物柱样中正构烷烃初探. 湖泊科学, 2003,15( 3 ):200-204.

[9] 姚书春,李世杰. 巢湖富营养化过程的沉积记录. 沉积学报,2004,22( 2 ):343 - 347.

[10] 王苏民,窦鸿身主编. 中国湖泊志. 北京:科学出版社,1998:230-235.

[ 11 ] 郭 斌,朱日祥,白立新等. 黄土沉积物的岩石磁学特征与土壤化作用的关系. 中国科学( D 辑 ),2001, 31( 5 ):377 - 386.

[ 12 ] Oldfield F, Yu L. The influence of particles size variations on the magnetic properties of sediments from the northeastern Irish Sea. Sedimentology, 1994,41:1093 - 1108.

[ 13 ] Maher B A, Thompson R. Mineral magnetic record of the Chinese loess and paleosols. Geology, 1991, 19: $3-6$.

[14] 张卫国, 俞立中. 长江口潮滩沉积物的磁学性质及其与粒度的关系. 中国科学( D 辑 ), 2002,32 (9): 783 - 792 . 